\title{
Physical fitness as a moderator of cognitive work capacity and fatigue onset under sustained combat-like operations
}

\author{
ROBERT J. PLEBAN and DEBRA A. THOMAS \\ U.S. Army Research Institute for the Behavioral and Social Sciences \\ Ft. Benning Field Unit, Ft. Benning, Georgia \\ and \\ HENRY L. THOMPSON \\ U.S. Army Command and General Staff College, Ft. Leavenworth, Kansas
}

\begin{abstract}
A study was devised to investigate the role of physical fitness in moderating both cognitive work capacity and fatigue onset under sustained combat operations. Sixteen male ROTC cadets were followed through a 2.5-day, Pre-Ranger Evaluation exercise. Prior to the start of the exercise, the cadets' overall level of physical fitness was assessed by using five fitness indices (Harvard Step Test, chin-ups, push-ups, sit-ups, and two-mile run). Cognitive performance and subjective measures of fatigue state were assessed at regular intervals before, during, and 1 day after the exercise. The results suggest that fitness may attenuate decrements in cognitive work capacity for certain tasks requiring prolonged mental effort, particularly as the cumulative effects of sleep loss and other stressors begin to mount. Similarly, the results suggest that as overall stress levels increase, fitness may have a beneficial effect in moderating fatigue rate. Fitness did not significantly enhance the recovery process with respect to cognitive work capacity, and actually appeared to hinder recovery from fatigue.
\end{abstract}

Recent studies (e.g., Angus \& Heslegrave, 1983; Haslam, 1978; Haslam, Allnutt, Worsley, Dunn, Abraham, Few, Labuc, \& Lawrence, 1977; Opstad, Ekanger, Nummestad, \& Raabe, 1978) concerned with the effects of sustained military operations on performance have indicated that the capacity to sustain intellectual and cognitive effort begins to deteriorate after 1 night of total or partial sleep deprivation, with noticeable degradation occurring in many instances following $48 \mathrm{~h}$ of sleep loss/deficit. The ability of the individual to successfully endure the demands imposed by high-intensity continuous operations depends to a large extent on the development of effective tactics and strategies for prolonging efficient performance, particularly on tasks that have a large cognitive or vigilance component.

Three of the four studies noted above (Haslam, 1978; Haslam et al., 1977; Opstad et al., 1978) showed that introducing small amounts of sleep (1.5-4 h) per $24 \mathrm{~h}$ is quite effective, relative to no sleep, in either reviving per-

The authors would like to thank Vincent M. O'Donnell, Lloyd M. Crumley, Ralph R. Canter, Harvey Babkoff, and Helen C. Sing for their valuable comments on a previous draft of this paper. Correspondence and requests for reprints should be addressed to: Robert J. Pleban, Walter Reed Army Institute of Research, Department of Behavioral Biology, Forest Glen Annex, Building 189, Washington, DC 20307-5100. formance or reducing the rate of performance decline on cognitive tasks.

However, as Kopstein, Siegal, Ozkaptan, Dyer, Conn, Slifer, and Caviness (1982) noted, the ultimate success of such tactics depends on how well prepared the individual/unit is to engage in sustained operations. They suggest a number of long-term precombat strategies for countering the negative effects of sustained operations on performance, such as cross-training, intense physical conditioning, practice while fatigued, etc.

A recent report by Hegge (1981) suggested that physical fitness may play a particularly valuable role in slowing the rate of performance decline during sustained combat operations. In general (Hegge, 1981), the greater the aerobic and anaerobic capacities of the individual are, the lower the relative rate of resource utilization at work and the higher the relative rate of resource recovery with rest. Although these relationships have been thought to hold mainly for tasks involving muscular effort, there is some reason to believe that they influence cognitive work capacity as well.

For example, Hammerton and Tickner (1968) exposed subjects, classified as either fit or ultrafit, to $400 \mathrm{sec}$ of intense physical exercise. After completing the exercise, subjects' performance on a skilled visual motor task of 
either moderate or great difficulty was assessed. Hammerton and Tickner found that fit subjects showed no exercise-associated decrement in performance on the moderately difficult task but a marked decrement on the extremely difficult task. In contrast, ultrafit subjects showed no decrement on the more difficult task.

\section{Objective}

The objective of the study reported here was to examine in more detail the potential moderating effects of physical fitness on cognitive work capacity and fatigue onset under sustained combat-like operations.

\section{METHOD}

\section{Subjects}

Sixteen male Reserve Officer Training Corps (ROTC) cadets, approximately 21 years of age, from the University of Georgia and Armstrong State College, participated in the study. Of these 16 cadets, 12 had applied for Ranger School in lieu of ROTC Advanced Camp for the coming summer. All cadets received approval to take part in the Pre-Ranger Evaluation exercise described in the Procedure section. During the course of the exercise, 1 cadet was injured and could not continue, and 3 cadets did not complete the last day of testing because of scheduling conflicts. Consequently, most of the data analyses were based on 12 subjects.

\section{Materials}

Cognitive performance was assessed using three paperand-pencil tests: (1) Logical Reasoning Test (Baddeley, 1968); (2) Map-Plotting Test (Haslam et al., 1977); and (3) Encoding-Decoding Test (Haslam et al., 1977).

The Logical Reasoning Test, developed by Baddeley (1968), is an exercise in transformational grammar. The modified version of this test (used by Haslam et al., 1977) consists of 16 short sentences of the following type: "A does not come before B." Each sentence is followed by the letters $A B$ or $B A$, and the individual is required to indicate by a check whether each of the statements is true of false. A time limit of $1 \mathrm{~min}$ was used.

The Map-Plotting Test is similar to one used by Haslam et al. (1977). It consists of a blank grid of $2045 / 8$-in. (approximately) squares with two-digit coordinates along the two (north and east) axes. Eighteen six-figure coordinates were to be plotted on the grid. These were listed on a separate sheet of paper. A 3-min time limit was imposed.

The Encoding-Decoding Test is a modified version of the one used by Haslam et al. (1977). It consists of 15 six-figure grid coordinates and 15 bigrams (e.g., BCFK) that have to be encoded and decoded, respectively, using a code strip printed across the top of the page. The time allotted for this test was $6 \mathrm{~min}$.

Since cognitive functioning was to be assessed on seven separate occasions (once a day), seven versions of each of the three cognitive tests were constructed to avoid possible learning of the materials.
Subjective measures of fatigue were assessed using three paper-and-pencil instruments. Fatigue was assessed using the Tiredness Scale (Haslam, 1978), the Stanford Sleepiness Scale (Hoddes, Zarcone, Smythe, Phillips, \& Dement, 1973), and the Mood Questionnaire-Fatigue subscale (Ryman, Biersner, \& La Rocco, 1974).

The Tiredness Scale is a 25-point scale with endpoints ranging from very fresh to very tired. The individual checks the point on the scale that best indicates his or her present level of tiredness. The Stanford Sleepiness Scale is a seven-statement inventory describing increasing stages of tiredness (e.g., feeling active and vital; alert-wide awake/almost in a dream; will be asleep soon; lost struggle to remain awake). The individual checks the statement that most closely corresponds to his or her present level of sleepiness. The Fatigue subscale of the Mood questionnaire consists of a five-adjective list (weary, lazy, drowsy, sluggish, and inactive). Each adjective is rated on a scale from 1 (not at all) to 3 (mostly or generally).

The cognitive and subjective measures (with the exception of map-plotting) have been shown to be sensitive to a variety of stressors, including sleep loss, physical work load, and psychological stress (Haslam, 1978; Haslam et al., 1977; Opstad et al., 1978). Although map-plotting was not a sensitive index of cognitive performance in the Haslam et al. (1977) study, it was included in the present study because of its perceived relevance to the Ranger exercise.

\section{Procedure}

The study covered a 7-day period divided into three phases: Days 1-3 (control), Days 4-6 (experimental), and Day 7 (recovery). On Day 1, cadets were briefed about the general purpose of the study. Starting on Day 1 and on each day thereafter, at approximately $1600 \mathrm{~h}$, cadets were administered the Logical Reasoning, Map-Plotting, and Encoding-Decoding tests, in that order. The specific versions of the three tests presented to the cadets were randomized. Prior to the start of each test in each testing period, the experimenter read aloud the specific instructions for that instrument to ensure that the cadets knew exactly what they were to do. Testing took place indoors during the control and recovery periods and on the 1st day of the experimental period (Day 4). Testing for the last 2 days of the experimental period was performed outdoors in the training area.

The purpose of the control period was to familiarize the cadets with the tests and to provide them with enough practice that performance would be relatively stable going into the Pre-Ranger Evaluation (PRE) exercise (Bittner, Jones, Carter, Shannon, Chatfield, \& Kennedy, 1981).

Beginning on Day 4 at $1600,{ }^{1}$ both performance and fatigue measures were collected. Fatigue was assessed at 4-h intervals throughout the experimental period and once during the recovery period (at $1600 \mathrm{~h}$ ) preceding the last series of cognitive tests. (Only fatigue ratings taken at $1600 \mathrm{~h}$ each day were utilized in this study, since it was not possible to obtain ratings for the other time intervals 
during the land navigation part of the exercise on Day 6.) At approximately $1730 \mathrm{~h}$ on Day 4, the cadets were given a physical fitness test that included five measures administered in the following order: Harvard Step Test, chin-ups, push-ups, sit-ups, and two-mile run.

The PRE exercise formally began at $1900 \mathrm{~h}$ on Day 4 . The purpose of the PRE exercise was to provide a comprehensive evaluation of Ranger applicants in a relatively short ( 2.5 days) but physically and mentally demanding exercise and to give applicants some insight into the magnitude of the stress involved in Ranger operations. This exercise was regarded as ideal, since it involved some of the key characteristics of a sustained operation (i.e., psychological stress, sleep loss, sustained activity, and caloric deficit).

At $1900 \mathrm{~h}$ (on Day 4), the cadets received their operations order. For the next 2 days (Days 5 and 6), as part of the PRE exercise, cadets participated in patrol missions, conducted raids and ambushes, and were tested on various military skills (e.g., first aid, weapons, and land navigation ability) at a training area. The cadets slept for approximately $2 \mathrm{~h}$ on Day $5(2200-2400 \mathrm{~h})$; they were restricted to one meal (canned Army $\mathrm{C}$ ration) per day and were kept physically active for most of the exercise. An attempt was made to enhance psychological stress by waiting until the last possible moment before informing the cadets of what their next task or mission entailed.

The PRE exercise ended on Day 6 at $1600 \mathrm{~h}$. Cadets were then returned to the ROTC building on campus. After approximately $24 \mathrm{~h}$ of sleep/rest, the cadets were tested for the final time (Day 7). Cognitive performance and subjective measures of fatigue were obtained. Once this final phase had been completed, the cadets were interviewed.

\section{RESULTS}

\section{The Fitness Construct}

The five separate fitness indices (chin-ups, push-ups, sit-ups, two-mile run, and pulse rate as determined from the Harvard Step Test) were combined to form a single fitness composite score. This was accomplished by first transforming the raw scores obtained for each of the five measures into $\mathrm{Z}$ scores. The $\mathrm{Z}$ scores were converted into percentiles based on the percentage of the total area of the normal curve each $\mathrm{Z}$ score represented. The individual percentiles were then added and divided by 5 (number of fitness measures) to get an average fitness rating for each cadet. $^{2}$

\section{Method of Analysis}

The primary focus of this study was directed toward examining the relationships between physical fitness and changes in both cognitive performance and fatigue state from baseline (Day 4) across time. Consequently, it was necessary to select a data-analytic strategy that would provide a relatively sensitive index of change.

Simply computing raw change scores (i.e., subtracting the prescore from the postscore) and relating these scores to fitness levels was ruled unacceptable as a data-analytic strategy for several reasons. First, raw or simple change scores are generally more unreliable than the variables being differenced. The greater unreliability of change scores serves to attenuate the correlation with other variables (Cohen \& Cohen, 1975; Hummel-Rossi \& Weinberg, 1975). Second, and more fundamental to the issue of change, is the necessary dependence of change scores on prescores. This dependence can further distort the relationship of change to other variables, since, in general, the raw change score contains unwanted variance from the prescore. This dependency operates regardless of the unreliability problem noted above (Cohen \& Cohen, 1975).

One solution, proposed by Cohen and Cohen (1975), for minimizing the biasing effects of the prescore in assessing change is to treat the prescore as a covariate and partial out, using partial correlation procedures, the variance from both the postscore and the research variable attributable to the prescore. Since the impact of the prescore is more precisely controlled for with this method, the resulting partial correlation will provide a less distorted index of the relationship between change and the treatment/subject variable of interest. See Cohen and Cohen (1975) for a more detailed description of the use of partial correlation procedures in the assessment of change.

For the present analysis, partial correlations were computed between fitness level and cognitive performance for both number attempted and number correct and between fitness level and fatigue ratings on Days 5, 6, and 7, with initial differences being controlled for on Day 4, the base day.

\section{Performance Decrement and Physical Fitness}

For this study, positive correlations were indicative of less performance decrement with increasing levels of fitness, whereas negative correlations were indicative of greater performance decrement with increasing fitness. It was expected that smaller performance decrements would be associated with higher levels of fitness.

The strongest support for this hypothesis occurred on Day 6 for encoding-decoding: number correct partial $r$ $=.50$, and number attempted partial $\mathrm{r}=.59, \mathrm{p}<.05$. The partial correlations for Days 5 and 7 were low (.23 to .33), but in the predicted direction. For the remaining two cognitive tasks (logical reasoning and map-plotting), the partial correlations obtained were either close to .00 or were negative.

\section{Fatigue Level and Physical Fitness}

With regard to the relationship between fatigue level and physical fitness over time, positive correlations were indicative of increasing amounts of fatigue with increasing levels of fitness, whereas negative correlations were indicative of less fatigue buildup over increasing levels of fitness. It was expected that fitness would attenuate the projected rise in fatigue over time and facilitate the recovery process.

Negative partial correlations between fitness and fatigue 
level were obtained for Day 6 only, with the correlation between fitness and the Fatigue subscale reaching statistical significance (partial $\mathrm{r}=-.57, \mathrm{p}<.05$ ).

\section{DISCUSSION}

Earlier reports (Kopstein et al., 1982; Hegge, 1981; Hammerton \& Tickner, 1968) suggested that physical fitness might play an important role in combating the adverse effects of sustained combat operations on cognitive work capacity and fatigue onset by slowing the rate of performance decline and the accompanying buildup of fatigue. The evidence from this study, although not conclusive, indicates that the relationships among physical fitness, cognitive work capacity, and fatigue level may be more complex than were originally hypothesized.

\section{Cognitive Work Capacity and Physical Fitness}

With respect to cognitive work capacity, the pattern of correlations obtained on the Encoding-Decoding task provided the strongest and most consistent support for the notion that fitness would be an effective moderator of performance decrement over time. The data seem to indicate that fitness may begin to assert its influence when the cognitive task or activity becomes more sustained in nature (e.g., $6 \mathrm{~min}$ or longer) and as the cumulative effects of sleep loss and other stressors begin to mount (i.e., last day in the field).

Task difficulty also may have contributed to the pattern of results that was obtained. Anecdotal reports from the cadets indicated that the Encoding-Decoding Task was the most difficult of the three cognitive tasks, as well as the longest. The importance of task difficulty as a moderating variable received some empirical support from the earlier findings of Hammerton and Tickner (1968), who found less performance decrement with increasing levels of fitness, but only for the more difficult cognitive task.

The results from the Map-Plotting Task are more difficult to explain. Four of the six partial correlations ranged from -.40 to -.84 , suggesting a fairly strong (although not statistically significant) inverse relationship between fitness level and performance decrement. Task and/or subject characteristics may have affected the pattern of results obtained.

Fitness did not appear to play a strong role in the recovery process (Day 7) with respect to cognitive functioning. Four of the six partial correlations between performance (decrement) and fitness level at Day 7 were in the predicted direction but not statistically significant. This could be attributed to low statistical power (Cohen, 1977) and a relatively long (24-h) recovery period.

\section{Fatigue Level and Physical Fitness}

The negative partial correlations between fitness and each of the three fatigue measures at Day 6 suggested that, as overall stress levels increase, fitness may have a beneficial effect in moderating fatigue rate. However, fitness did not facilitate the reduction of fatigue over the $24-\mathrm{h}$ sleep/rest recovery period. In fact, on Days 5 and 7, the more fit cadets reported the greatest levels of fatigue.

The shift in the direction of the correlations from positive on Day 5 to negative on Day 6 and back to positive on Day 7 is difficult to explain, particularly given the apparent magnitude of the shifts that were obtained (67 to 105 units). A modified replication of the present study is needed to clarify the unexpected patterns of results that were obtained on both the cognitive and fatigue measures.

\section{REFERENCES}

Angus, R. G., \& Heslegrave, R. J. (1983). The effects of sleep loss and sustained mental work: Implications for command and control performance. In Proceedings of the 24th Defense Research Group Seminar (Vol. 1). Downsview, Ontario, Canada: Defence and Civil Institute of Environmental Medicine.

BADDELEY, A. D. (1968). A 3-minute reasoning test based on grammatical transformations. Psychonomic Science, 10, 341-342.

Bittner, A. C., Jones, M. B., Carter, R. C., Shannon, R. H., Chatfield, D. C., Kennedy, R. S. (1981). Statistical issues in performance testing: Collected papers (Reprint No. NBDL-81 R010). New Orleans, LA: U.S. Naval Biodynamics Laboratory.

COHEN, J. (1977). Statistical power analysis for the behavioral sciences. New York: Academic Press.

COHEN, J., \& COHEN, P. (1975). Applied multiple regression/correlation analysis for the behavioral sciences. Hillsdale, NJ: Erlbaum.

Hammerton, M., \& Tickner, A. H. (1968). Physical fitness and skilled work after exercise. Ergonomics, 11, 41-45.

HASLAM, D. R. (1978). The effect of continuous operations upon the military performance of the infantryman (Exercise "Early Call" II) (Report APRE No. 4/78). Farnborough, England: Army Personnel Research Establishment.

haslam, D. R., Allnutt, M. F., Worsley, D. E., Dunn, D., Abraham, P., Few, J., Labuc, S., \& LaWrence, D. J. (1977). The effect of continuous operations upon the military performance of the infantryman (Exercise "Early Call") (Report APRE No. 2/77). Farnborough, England: Army Personnel Research Establishment.

HeGGE, F. W. (1981). Control of human resources. In NATO Defense Research Group (Eds.), Study on human and biomedical aspects of the sustained operation. Washington, DC: Walter Reed Army Institute of Research.

Hoddes, E., Zarcone, V., Smythe, H., Phillips, R., \& Dement, W. (1973). Quantification of sleepiness: A new approach. Psychophysiology, 10, 431-436.

Hummel-Rossi, B., \& Weinberg, S. (1975). Practical guidelines in applying current theories to the measurement of change. Psychological Documents, 5, 226.

Kopstein, F., Siegel, A., Ozkaptan, H., Dyer, F., Conn, J., Sufer, W., \& CAVINESS, J. (1982). Soldier performance in continuous operations (Contract No. MDA903-81-C-0280). Wayne, PA: Applied Psychological Services, Inc.

Opstad, P. K., Ekanger, R., Nummestad, M., \& RaAbe, N. (1978). Performance, mood, and clinical symptoms in men exposed to prolonged, severe physical work and sleep deprivation. Aviation Space Environmental Medicine, 49, $1065-1073$.

Ryman, D. H., BIERSNer, R. J., \& LARocCo, J. M. (1974). Reliabilities and validities of the mood questionnaire. Psychological Reports, 35, 479-484.

\section{NOTES}

1. Testing the cadets at $1600 \mathrm{~h}$ each day was done to have the testing period fall within the upper range of the circadian cycle (for cognitive functioning) and because it was the most convenient time for testing the cadets in the field.

2 . These ratings were distributed over a fairly wide range (13th-86th percentile) along the fitness continuum. 